\title{
Mitogenome reconstruction of an endangered African seahorse from a Traditional Chinese Medicine market was based on a misidentification
}

\author{
Peter R. Teske \\ Centre for Ecological Genomics and Wildlife Conservation, Department of Zoology, University \\ of Johannesburg, Auckland Park 2006, South Africa
}

\begin{abstract}
The recently published complete mitochondrial genome of the endangered Knysna seahorse, Hippocampus capensis Boulenger, 1900, was based on a specimen obtained from a Traditional Chinese Medicine market. As H. capensis is endemic to temperate South Africa and exceptionally rare, illegal trade to supply Asian markets would constitute a considerable extinction risk. I investigated the phylogenetic placement of the Chinese specimen using mitochondrial DNA control region and cytochrome $b$ sequences from the $H$. capensis mitogenome among corresponding published sequences of $H$. capensis and a number of closely related seahorse species. The Chinese specimen was distinct from $H$. capensis and instead clustered with $H$. casscsio, a seahorse that was recently described from the South China Sea. The sequences of $H$. casscsio clustered randomly among those of specimens identified as $H$. fuscus, a species whose taxonomic validity is disputed, and which is considered to be a synonym of the widespread Indo-Pacific seahorse H. kuda. Given that the morphological identification of seahorses is difficult, it is recommended that the publication of new species descriptions and genomic resources be preceded by a comprehensive comparison with the available molecular data. The taxonomy of seahorses is far from resolved, and cutting-edge molecular studies will not improve this situation if they do not take existing information into consideration.
\end{abstract}

Keywords: Hippocampus; Mitochondrial DNA; Mitogenome; Seahorse; Taxonomy; Traditional Chinese Medicine

\section{Introduction}

Seahorses (genus Hippocampus Rafinesque, 1810) are teleost fishes from the family Syngnathidae which have a highly specialized morphology that includes an upright posture, a prehensile tail, fused jaws, an exoskeleton and a male brood pouch. While these unique morphological features make seahorses easy to recognize, distinguishing between different species is difficult because of considerable morphological variation that is affected by factors such as age, gender and the environment (Lourie et al. 2016). As a result, a large number of synonyms exist, and the number of species accepted as valid in recent taxonomic treatments have ranged from 23 to 83 species (Lourie et al. 2016). 
Molecular methods represent a useful independent means of confirming species status that requires little taxonomic expertise (Hebert et al. 2003), and such methods are now often used to support the distinctness of newly described species from morphologically similar seahorses (González et al. 2014; Zhang et al. 2016; Short et al. 2020), or challenge the validity of new species descriptions (Teske et al. 2007). While most studies have used the DNA barcoding marker for animals, the mitochondrial DNA cytochrome $c$ oxidase subunit I gene (COI or cox1), advances in DNA sequencing technology have now made it possible to sequence the entire mitochondrial genome (or mitogenome), and several seahorse mitogenomes have recently been published (Zhang et al. 2015; Wang et al. 2016, 2019b; Chen et al. 2018; Ge et al. 2018; Lai et al. 2019; Jahari et al. 2020). The increased level of resolution provided by mitogenomes can be expected to significantly improve taxonomic resolution in cases where DNA barcoding provides insufficient information for distinguishing closely related species.

One of the recently published seahorse mitogenomes, that of the endangered Knysna seahorse, Hippocampus capensis Boulenger, 1900 (Ge et al. 2018), was reconstructed using DNA of a specimen obtained from the Bozhou Chinese herbal medicine market (Bozhou, Anhui Province, PR China). The import of African seahorses for Traditional Chinese Medicine (TCM) markets in Asia is very common, and has resulted in a serious depletion of local stocks (T. Mkare, KMFRI, Mombasa, Kenya and I. da Silva, Universidade Lúrio, Pemba, Mozambique, pers. comm.). For example, the mitogenome of the East African H. camelopardalis was generated using a specimen from the same TCM market in Bozhou (Lai et al. 2019), and both the West African seahorse $H$. algiricus and the South Africa $H$. capensis were reported from a Taiwanese TCM market (Chang et al. 2013). The sale of $H$. capensis at these markets is a concern because this species is endangered, and endemic to three estuaries on the temperate south coast of South Africa (Teske et al. 2003; Mkare et al. 2017). The illegal collection of these seahorses for TCM markets can be expected to rapidly drive this exceptionally rare species to extinction.

The morphological identification of the Chinese specimen was relatively vague, and based on the absence of a coronet, a short snout and the absence of spines on the body. A comparison of the image provided in the mitogenome announcement with $H$. capensis suggested a questionable identification, with the difference in snout length being the most obvious morphological discrepancy (Teske et al. 2005). In contrast, the specimen of $H$. capensis from a TCM in Taiwan (Chang et al. 2013) resembles South African H. capensis more closely. To confirm the suspected distinctness of the Chinese seahorse used for the mitogenome reconstruction from the South African $H$. capensis, I compared its DNA sequences of partial mitochondrial control region and cytochrome $b$ sequences with those of $H$. capensis and a number of closely related species. The reason for selecting these two markers is that they are available from a comparatively large number of species and locations.

\section{Methods}

Sequences were downloaded from GenBank and aligned in MEGA7 (Kumar et al. 2016) using the ClustalW alignment tool (Thompson et al. 2002). Consensus sequences 402 bp (control 
region) and $836 \mathrm{bp}$ (cytochrome $b$ ) in length that were present in most sequences were then used to reconstruct phylogenetic trees using the neighbour-joining algorithm (Saitou and Nei 1987) in MEGA7. In both cases, the Tamura 3-parameter model (Tamura 1992) with gamma distribution was identified as the most suitable model of nucleotide evolution using the Bayesian Information Criterion (Schwarz 1978). Pairwise deletion of missing data was applied, and nodal support was based on 1000 bootstrap replications (Felsenstein 1985).

\section{Results and Discussion}

Both phylogenetic trees recovered previously published Hippocampus capensis sequences (some of which I collected myself and can thus vouch for their authenticity) as well supported monophyletic clusters. The Chinese specimen used for the mitogenome reconstruction of $H$. capensis was not part of these clusters, but was grouped with sequences from the recently described H. casscsio Zhang, Qi, Wang \& Lin, 2016, specimens identified as H. fuscus Rüppell, 1838 from the Mediterranean, the Red Sea and, in the case of cytochrome $b$ data, the Arabian Sea, as well as $H$. kuda Bleeker, 1852. 


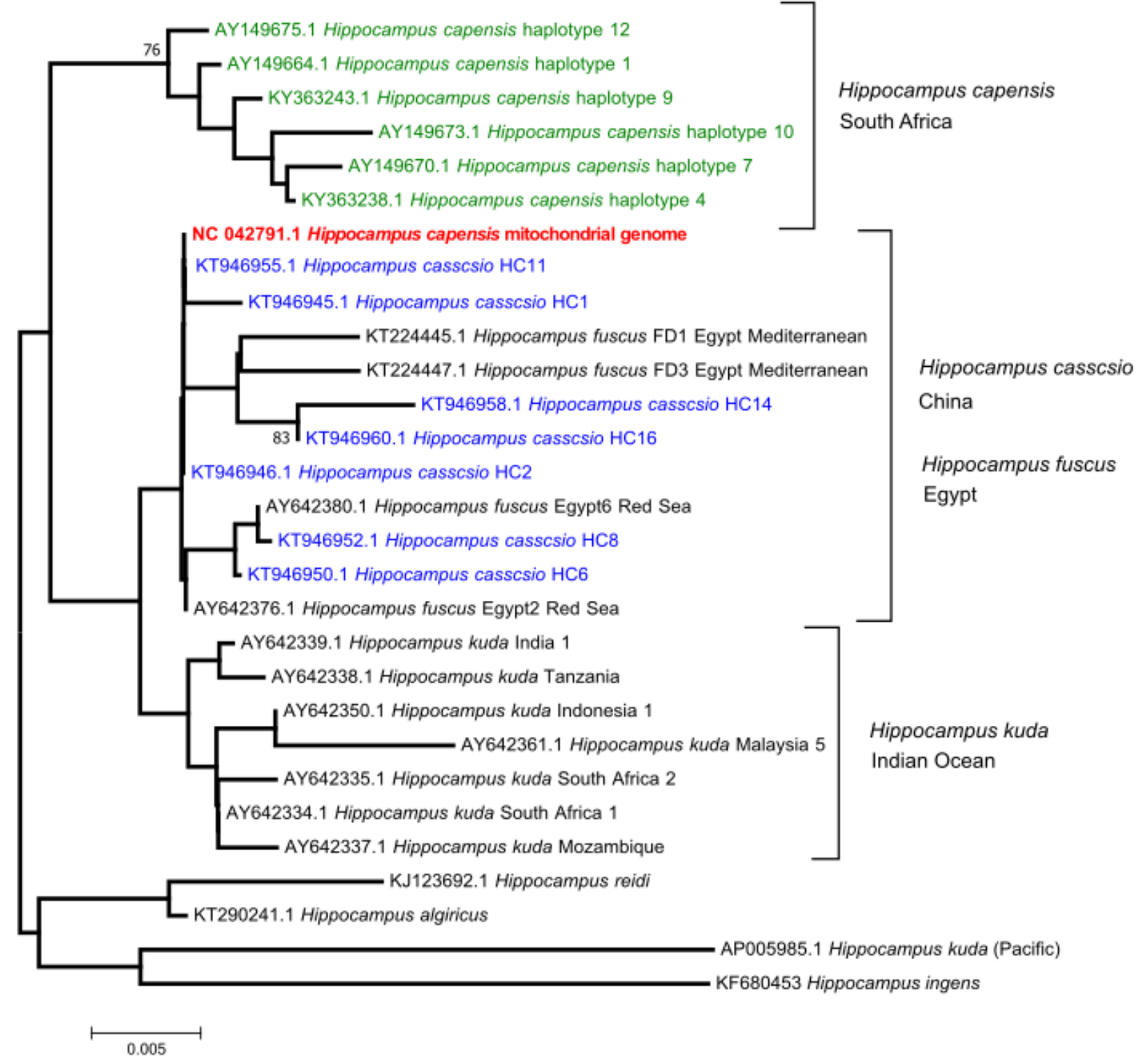

Fig. 1. Neighbour-joining tree of $406 \mathrm{bp}$ of control region sequences from Hippocampus capensis (South Africa), the Chinese specimen used for the mitogenome reconstruction of $H$. capensis (red), the recently described H. casscsio from the South China Sea, and a selection of closely related species. Bootstrap values $>75 \%$ are shown next to some nodes. 


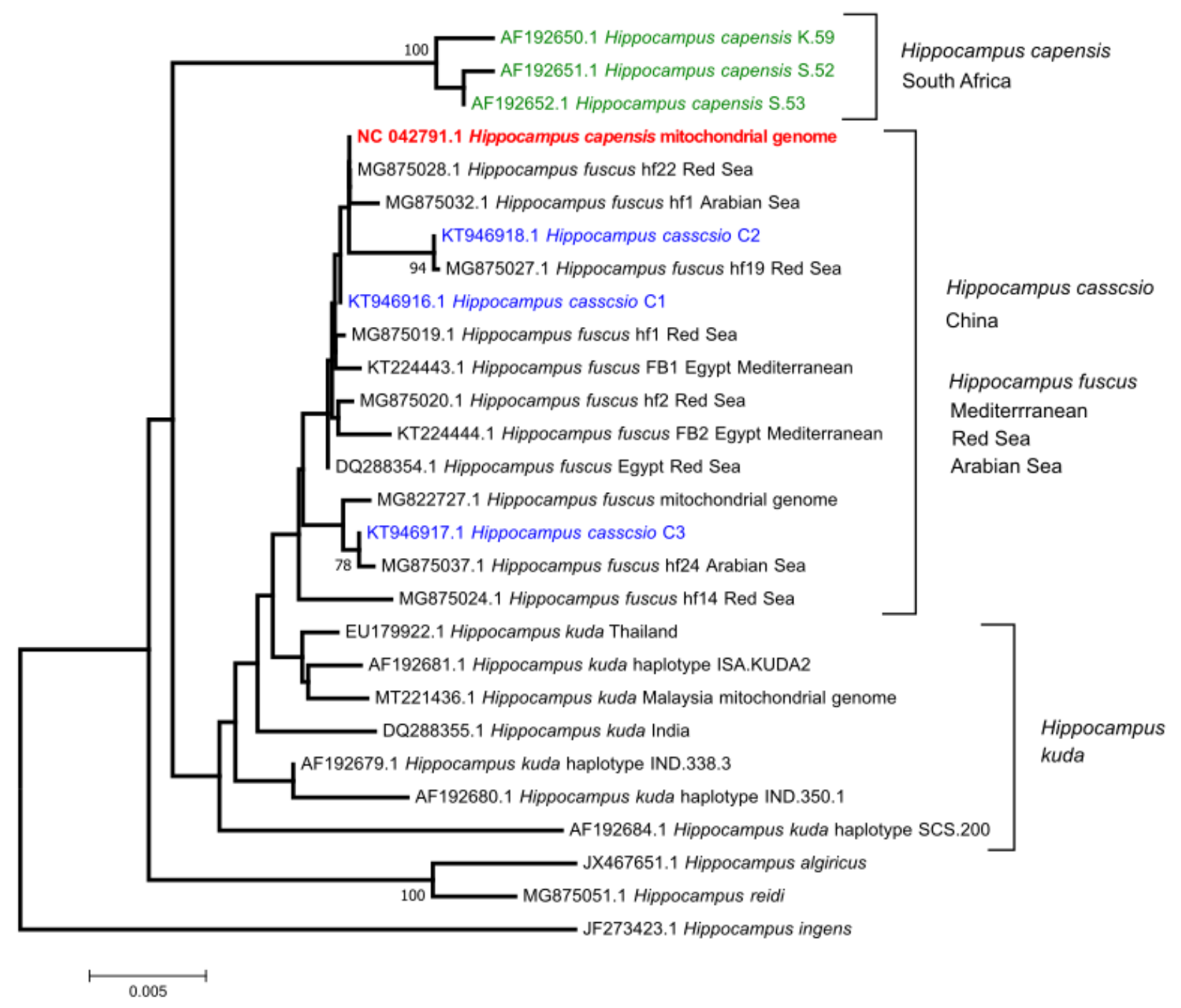

Fig. 2. Neighbour-joining tree of $836 \mathrm{bp}$ of control region sequences from Hippocampus capensis (South Africa), the Chinese specimen used for the mitogenome reconstruction of $H$. capensis (red), the recently described H. casscsio from the South China Sea, and a selection of closely related species. Bootstrap values $>75 \%$ are shown next to some nodes. 
These results confirm that the Chinese specimen was misidentified. The portion of the mitochondrial control region of this specimen is identical to one of the sequences of $\mathrm{H}$. casscsio, from Beibu Bay, South China Sea (Zhang et al. 2016), whereas its partial cytochrome $b$ sequence is identical to that of a specimen of H. fuscus from Safaga on the Red Sea coast of Egypt (Wang et al. 2019a). All Chinese seahorses clustered randomly among sequences of $H$. fuscus, a species name that was considered to be a synonym of $H$. kuda in a recent taxonomic revision of seahorses (Lourie et al. 2016). Bootstrap support for its distinctness was low in both trees, but in the control region tree all $H$. fuscus and $H$. casscsio sequences formed a sister clade to $H$. kuda, and in the cytochrome $b$ tree they formed a monophyletic cluster that was nested within a more diverse $H$. kuda cluster. This, and the fact that $H$. fuscus seems to be morphologically distinct from $H$. kuda because of the absence of a coronet (Teske et al. 2005), a trait that it shares with $H$. capensis (Teske et al. 2005) and H. casscsio (Zhang et al. 2016), suggests that it is worthy of renewed taxonomic attention. Incomplete lineage sorting between the Arabian Sea and the South China Sea indicates that this species may be represented in the vast, unsampled region separating these two locations. Due to the relatively low information content of the two mitochondrial markers used here, it cannot be ruled out that $H$. casscsio and $H$. fuscus are distinct species that have diverged very recently. However, until this is confirmed with high-resolution genomic data (Teske et al. 2019), the species status of $H$. casscsio should be rejected.

In conclusion, while the generation of mitogenomic data for seahorses can be expected to clarify taxonomic relationships between problematic species, it is important that such resources are compared with as many previously generated sequences as possible prior to publication. In this particular case, the fact that the phylogenetic trees presented in mitogenome announcements typically only include previously published complete mitogenomes (Jooste et al. 2019), to the exclusion of shorter fragments from more closely related species, prevented the discovery of the misidentification of the specimen on which the problematic $H$. capensis mitogenome was based. Moreoever, while the incorporation of genetic data into the species description of H. casscsio is commendable, the fact that the new species was only compared with Chinese seahorses, while published data from more closely related species were ignored, likely resulted in nothing more than the description of yet another synonym. This is a problematic trend that we highlighted over a decade ago (Teske et al. 2007). As such, failure to integrate existing molecular data will merely exacerbate the already confusing situation concerning the taxonomy of the genus Hippocampus.

Acknowledgements. I am grateful to R.A. Sreepada (National Institute of Oceanography, India) for pointing out the problematic mitogenome of Hippocampus capensis.

\section{References}

Chang C-H, Jang-Liaw N-H, Lin Y-S, et al (2013) Authenticating the use of dried seahorses in the traditional Chinese medicine market in Taiwan using molecular forensics. J Food Drug Anal 21:310-316. https://doi.org/10.1016/j.jfda.2013.07.010

Chen M, Zhu L, Chen J, et al (2018) The complete mitochondrial genome of the short snouted seahorse Hippocampus hippocampus Linnaeus 1758 (Syngnathiformes: Syngnathidae) and its 
phylogenetic implications. Conserv Genet Resour 10:783-787. https://doi.org/10.1007/s12686017-0930-y

Felsenstein J (1985) Confidence limits on phylogenies: an approach using the bootstrap. Evol Int J Org Evol 39:783-791. https://doi.org/10.1111/j.1558-5646.1985.tb00420.x

Ge Y, Zhu L, Chen M, et al (2018) Complete mitochondrial genome sequence for the endangered Knysna seahorse Hippocampus capensis Boulenger 1900. Conserv Genet Resour 10:461-465. https://doi.org/10.1007/s12686-017-0849-3

González R, Dinghi P, Corio C, et al (2014) Genetic evidence and new morphometric data as essential tools to identify the Patagonian seahorse Hippocampus patagonicus (Pisces, Syngnathidae). J Fish Biol 84:459-474. https://doi.org/10.1111/jfb.12299

Hebert PDN, Cywinska A, Ball SL, deWaard JR (2003) Biological identifications through DNA barcodes. Proc Biol Sci 270:313-321. https://doi.org/10.1098/rspb.2002.2218

Jahari PNS, Abdul Malik NF, Shamsir MS, et al (2020) The first complete mitochondrial genome data of Hippocampus kuda originating from Malaysia. Data Brief 31:105721. https://doi.org/10.1016/j.dib.2020.105721

Jooste CM, Emami-Khoyi A, Gan HM, et al (2019) The complete mitochondrial genome of Africa's largest freshwater copepod, Lovenula raynerae. Mitochondrial DNA Part B 4:725-727. https://doi.org/10.1080/23802359.2018.1555013

Kumar S, Stecher G, Tamura K (2016) MEGA7: molecular evolutionary genetics analysis version 7.0 for bigger datasets. Mol Biol Evol 33:1870-1874. https://doi.org/10.1093/molbev/msw054

Lai M, Sun S, Chen J, et al (2019) Complete mitochondrial genome sequence for the seahorse adulteration Hippocampus camelopardalis Bianconi 1854. Mitochondrial DNA Part B 4:432433. https://doi.org/10.1080/23802359.2018.1551081

Lourie SA, Pollom RA, Foster SJ (2016) A global revision of the seahorses Hippocampus Rafinesque 1810 (Actinopterygii: Syngnathiformes): taxonomy and biogeography with recommendations for further research. Zootaxa 4146:1-66. https://doi.org/10.11646/zootaxa.4146.1.1

Mkare TK, van Vuuren BJ, Teske PR (2017) Conservation implications of significant population differentiation in an endangered estuarine seahorse. Biodivers Conserv 1-19. https://doi.org/10.1007/s10531-017-1300-5

Saitou N, Nei M (1987) The neighbor-joining method: a new method for reconstructing phylogenetic trees. Mol Biol Evol 4:406-425. https://doi.org/10.1093/oxfordjournals.molbev.a040454

Schwarz GE (1978) Estimating the dimension of a model. Ann Stat 6:461-464

Short G, Claassens L, Smith R, et al (2020) Hippocampus nalu, a new species of pygmy seahorse from South Africa, and the first record of a pygmy seahorse from the Indian Ocean (Teleostei, Syngnathidae). ZooKeys 934:141-156. https://doi.org/10.3897/zookeys.934.50924 
Tamura K (1992) Estimation of the number of nucleotide substitutions when there are strong transitiontransversion and G+C-content biases. Mol Biol Evol 9:678-687. https://doi.org/10.1093/oxfordjournals.molbev.a040752

Teske PR, Cherry MI, Matthee CA (2003) Population genetics of the endangered Knysna seahorse, Hippocampus capensis. Mol Ecol 12:1703-1715. https://doi.org/10.1046/j.1365294X.2003.01852.x

Teske PR, Hamilton H, Palsbøll PJ, et al (2005) Molecular evidence for long-distance colonization in an Indo-Pacific seahorse lineage. Mar Ecol Prog Ser 286:249-260. https://doi.org/10.3354/meps286249

Teske PR, Lourie SA, Matthee CA, Green DM (2007) Hippocampus queenslandicus Horne, 2001 - a new seahorse species or yet another synonym? Aust J Zool 55:139-145. https://doi.org/10.1071/ZO07021

Teske PR, Sandoval-Castillo J, Golla TR, et al (2019) Thermal selection as a driver of marine ecological speciation. Proc R Soc B Biol Sci 286:20182023. https://doi.org/10.1098/rspb.2018.2023

Thompson JD, Gibson TJ, Higgins DG (2002) Multiple sequence alignment using ClustalW and ClustalX. Curr Protoc Bioinforma Chapter 2:Unit 2.3. https://doi.org/10.1002/0471250953.bi0203s00

Wang X, Han X, Zhang Y, et al (2019a) Phylogenetic analysis and genetic structure of the seahorse, Hippocampus fuscus from the Arabian and Red Sea based on mitochondrial DNA sequences. Mitochondrial DNA Part DNA Mapp Seq Anal 30:165-171. https://doi.org/10.1080/24701394.2018.1467410

Wang X, Zhang Y, Zhang H, et al (2016) Complete mitochondrial genome sequence of the longsnout seahorse Hippocampus reidi (Ginsburg, 1933; Gasterosteiformes: Syngnathidae). Mitochondrial DNA Part DNA Mapp Seq Anal 27:1401-1402. https://doi.org/10.3109/19401736.2014.947600

Wang X, Zhang Y, Zhang H, et al (2019b) Complete mitochondrial genomes of eight seahorses and pipefishes (Syngnathiformes: Syngnathidae): insight into the adaptive radiation of syngnathid fishes. BMC Evol Biol 19:119. https://doi.org/10.1186/s12862-019-1430-3

Zhang H, Zhang Y, Lin Q (2015) Complete mitochondrial genome of the pacific seahorse Hippocampus ingens Girard, 1858 (Gasterosteiformes: Syngnathidae). Mitochondrial DNA 26:755-756. https://doi.org/10.3109/19401736.2013.850680

Zhang Y-H, Qin G, Wang X, Lin Q (2016) A new species of seahorse (Teleostei: Syngnathidae) from the South China Sea. Zootaxa 4170:384-392. https://doi.org/10.11646/zootaxa.4170.2.11 